\title{
Consensus of Multi-Agent Systems Using Back-Tracking and History Following Algorithms
}

\author{
Yanumula V. Karteek*, Indrani Kar*, Somanath Majhi*
}

\begin{abstract}
This paper proposes two algorithms, namely "back-tracking" and "history following", to reach consensus in case of communication loss for a network of distributed agents with switching topologies. To reach consensus in distributed control, considered communication topology forms a strongly connected graph. The graph is no more strongly connected whenever an agent loses communication. Whenever an agent loses communication, the topology is no more strongly connected. The proposed back-tracking algorithm makes sure that the agent backtracks its position unless the communication is reestablished, and path is changed to reach consensus. In history following, the agents use their memory and move towards previous consensus point until the communication is regained. Upon regaining communication, a new consensus point is calculated depending on the current positions of the agents and they change their trajectories accordingly. Simulation results, for a network of six agents, show that when the agents follow the previous history, the average consensus time is less than that of back-tracking. However, situation may arise in history following where a false notion of reaching consensus makes one of the agents stop at a point near to the actual consensus point. An obstacle avoidance algorithm is integrated with the proposed algorithms to avoid collisions. Hardware implementation for a three robots system shows the effectiveness of the algorithms.
\end{abstract}

\section{Key Words}

Consensus; networked multi-agent system; switching topologies based on position; back-tracking; history following

\section{Introduction}

The consensus problem was conceptualized after the exploration of self-ordered biologically motivated particle motions by Vicsek et al. 1 in 1995. A survey of the literature in this area can be found in 2. When multiple robots or agents are used to perform a combined task, they are expected to cooperate with each other 3 . 5 . To behave cooperatively, all the agents must agree on a specific variable, be it position, velocity or something else. When they concur on a suitable variable or variables, the consensus is reached. Graph theory is popularly used to represent a network of agents with nodes and branches in a graph equivalent to agents and communication links among agents respectively [6. 7]. Saber and Murray [6] considered undirected networks with distributed linear and nonlinear consensus protocols to solve an average consensus problem. They also found out an upper bound on the tolerable fixed time-delay in the system. The same authors then analyzed the convergence for three different cases in 7]. Moreau 8 investigated on the stability of consensus algorithms and derived sufficient conditions to guarantee uniform exponential stability. Linear time varying systems with communication time-delays are considered in this work and a theoretical analysis was provided without any simulation results. Convergence of the topologies and performance are also addressed by them. Saber et al. [9], provided a review of various first order consensus algorithms with and without time-delays. Sufficient condition for convergence in case of higher order systems is given by Ren et. al [10]. However, communication time-delays are not considered by the analysis. A detailed study about time-delays in consensus protocols is presented in 11]. Second order leaderless autonomous multi-agents with time-varying communication delays that are connected by a directed graph are considered in 12 and the stability analysis is also provided. Directed graph with switching topologies and time-delays are considered in [13 for first order with extension to higher order systems to prove the boundedness. A necessary and sufficient condition for consensus without time-delays in high-order systems with an undirected fixed topology is presented in 14]. Consensus of higher order multi-agents with fixed time-delays in discrete time is considered in [15] using nearestneighbour rule. A completely different approach was discussed in 16 where a fuzzy sliding mode controller was designed

\footnotetext{
*Department of EEE, IIT Guwahati, Assam, India; email: yanumula@alumni.iitg.ac.in, \{indranik,smajhi\}@iitg.ac.in
} 
for consensus of multi-agent system without considering the time-delays. Hardware implementation of multi-robot system for transportation is presented by Ting et. al [17].

In this paper, a network of fifth order systems with switching topologies and time-delays is considered in which some of them are not strongly connected. Topology switching occurs in case of link loss and regain which is a very common phenomenon in any communication network. Presence of big obstacles like wall may cause loss of link and situation may arise when the link is never regained unless the position of the agent is changed. In such situations, positions of agents are closely related to switching of the communication topology. Two new algorithms called "back-tracking" and "historyfollowing" are proposed to efficiently handle such situations when the communication topology is not strongly connected mainly because of the positions of the agents. According to the proposed back-tracking algorithm, an agent backtracks its path if it loses communication with other agents and choose a new path to reach the consensus. However, in the history-following case, the agents use their memory to follow the previous consensus point once the communication is lost. A new consensus point is calculated after the communication is re-established and the network is strongly connected again. The group of $n$ agents and the underlying communication topology is represented by a directed graph with $n$ nodes and corresponding branches representing the communication topology. Branch weights of the directed graph are considered to be unity rather than non-uniform weights. The switching topologies based on position of agents are represented by corresponding directed graphs and adjacency matrices. Static obstacles are considered to be present in the paths of the agents which necessitates an obstacle avoidance algorithm to be incorporated with.

\section{System Model}

A group of $n$ robotic agents are considered with input and communication time-delays, network topology of the group of robotic agents is represented by a directed graph $G[7$. Then there exists a vertex set $V=\{1,2, \ldots, n\}$ representing nodes and an edge set $E=\{(i, j): i, j \in V\}$ representing branches. The edge set comprises of ordered pairs $(i, j)$ if node $i$ is sending data to node $j$, which are represented by branches in the directed graph. The directed graph $G$ has an adjacency matrix $A=\left(a_{j i}\right)_{n \times n}$ with $a_{i i}=0, \forall i \in V, a_{j i}=1$ if $(i, j) \in E$ and $a_{j i}=0$ otherwise. Communication time-delay among agents is represented by $g_{j} T$ and processing time-delay of each agent is represented by $g_{i} T$. The graph Laplacian matrix $L=\left(l_{i j}\right)_{n \times n}$ of the directed graph $G$ have the elements $l_{i j}=\sum_{i \neq j} a_{j i}$ and $l_{i j}=-a_{j i}, \forall i \neq j$. For a strongly connected graph, $L$ has a zero eigenvalue and $[1, \ldots ., 1]^{T} \in \Re^{n}$ is the corresponding eigenvector, rest of the eigenvalues have negative real parts.

\subsection{Simulation Model}

The $i^{t h}$ agent dynamics in a coordinate system positioned at $\left(x_{i}, y_{i}\right)$ with an orientation of $\phi$ radians is given by

$$
\begin{aligned}
& \dot{x}_{i}=v_{i} \cos \left(\phi_{i}\right) ; \quad \dot{y}_{i}=v_{i} \sin \left(\phi_{i}\right) \\
& \dot{\phi}_{i}=\omega_{i} ; \quad \dot{v}_{i}=u_{i 1} ; \quad \dot{\omega}_{i}=u_{i 2}
\end{aligned}
$$

where $v_{i} \in \Re^{m}$ and $\omega_{i} \in \Re^{m}$ are linear velocity and angular velocity of the agent, $\dot{v}_{i}$ and $\dot{\omega}_{i}$ denote linear acceleration and angular acceleration and $u_{i 1}, u_{i 2} \in \Re^{m}$ are corresponding control inputs.

$$
\begin{aligned}
& u_{i 1}=f_{1}\left(u_{i x}, u_{i y}, u_{i v}\right) \\
& u_{i 2}=f_{2}\left(u_{i x}, u_{i y}, u_{i v}\right)
\end{aligned}
$$

where

$$
\begin{aligned}
& u_{i x}=\frac{1}{\sum_{j=1}^{n} a_{j i}} \sum_{j=1}^{n} a_{j i}\left(x_{j}\left(\left(k-g_{j}\right) T\right)-x_{i}\left(\left(k-g_{i}\right) T\right)\right) \\
& u_{i y}=\frac{1}{\sum_{j=1}^{n} a_{j i}} \sum_{j=1}^{n} a_{j i}\left(y_{j}\left(\left(k-g_{j}\right) T\right)-y_{i}\left(\left(k-g_{i}\right) T\right)\right)
\end{aligned}
$$

are feedback inputs corresponding to the local interactions with respect to the agent.

$$
u_{i v}=v_{i}\left(\left(k-g_{i}\right) T\right)
$$

gives feedback information about velocity of the agent. $a_{i j}=a_{j i}$ for undirected graph.

It is assumed that position and velocity are constant in the interval $[k T,(k+1) T)$. Communication and input time delays are represented by $g_{j} T$ and $g_{i} T$ respectively. To emulate the situation where topology changes due to specific positions of the agents. The control laws in equation (2) $f_{1}\left(u_{i x}, u_{i y}, u_{i v}\right)$ and $f_{2}\left(u_{i x}, u_{i y}, u_{i v}\right)$ obey the following algorithmic steps. 
1. If step $=1$, the agents broadcast their positions without moving. If $1<$ step $\leq$ step $_{\min }$, the agents broadcast messages to incorporate time-delay in the system.

2. The heading of each agent is updated by the control law $f_{2}\left(u_{i x}, u_{i y}, u_{i v}\right)$ based on feedback from other agents when the agent is not inside consensus circle radius $c c r\left(\sqrt{u_{i x}{ }^{2}+u_{i y}{ }^{2}}>c c r\right)$ with center as average position of other agents.

3. $f_{1}\left(u_{i x}, u_{i y}, u_{i v}\right)= \pm a$ if $\sqrt{u_{i x^{2}}{ }^{2} u_{i y}{ }^{2}}>c c r$ and $u_{i v}<v_{\max }$ i.e., if an agent is not inside the consensus circle radius $c c r$, set acceleration as $\pm a$ until velocity of the agent reaches $v_{\max }$.

4. $f_{2}\left(u_{i x}, u_{i y}, u_{i v}\right)$ continuously updates the heading using obstacle avoidance algorithm in section-5.

5. Repeat above algorithmic steps until $\sqrt{u_{i x^{2}}+u_{i y}{ }^{2}} \geq c c r$.

6. When an agent loses communication with other agents, the agent is brought to halt by setting deceleration $d$ until velocity becomes zero using $f_{1}\left(u_{i x}, u_{i y}, u_{i v}\right)=-d$ if $v_{i}>0$.

7. When $\sqrt{u_{i x}{ }^{2}+u_{i y}{ }^{2}}<c c r, f_{1}\left(u_{i x}, u_{i y}, u_{i v}\right)=-d$ if $v_{i}>0$ and $f_{1}\left(u_{i x}, u_{i y}, u_{i v}\right)=0$ if $v_{i}=0$ i.e., the agent is brought to halt by decelerating until velocity becomes zero.

8. The simulation is continued till step $\max$ number of steps.

The parameters $s t e p_{\min }, \mathrm{step}_{\max }, c c r, a, d$ and $v_{\max }$ are to be chosen by the users based on the scenario. The above position based switching algorithm without back-tracking or history following(memoryless agents) is elaborated in a flow chart as shown in Fig. 2.

Control inputs $u_{i 1}, u_{i 2}$ in (2) are able to solve consensus problem if the graph is a spanning tree 12, 18. A graph becomes strongly connected when every node has a directed path to all the other nodes. Likewise, the graph is called a spanning tree when all nodes have a directed path from at least one node. In distributed control, the network need not be strongly connected to reach consensus 19 . Consensus can be ensured if the graph is a spanning tree for fixed topology or the union of all the topologies is a spanning tree for a periodic switching topologies [15]. However, in a spanning tree topology, non-root agents may not have information about other agents. This may lead to the agents not being able to decide on coming to halt or use back-tracking algorithm when the topology is not a spanning tree. To overcome the issue, we have considered strongly connected topology as a necessary condition.

If $x_{i} \rightarrow x_{j}, y_{i} \rightarrow y_{j}, \forall i \neq j$ and $\dot{x}_{i} \rightarrow 0, \dot{y}_{i} \rightarrow 0, \dot{\phi}_{i} \rightarrow 0, \forall i \in \Re^{n}, \forall j \in \Re^{n}$, then consensus is said to be reached in position for $n$ point masses. It can also be said that, consensus in position of $n$ robots is reached if, $x_{i} \rightarrow x_{i}{ }^{*}$ and $y_{i} \rightarrow y_{i}{ }^{*}$ and $\dot{x}_{i} \rightarrow 0, \dot{y}_{i} \rightarrow 0, \dot{\phi}_{i} \rightarrow 0$ where substituting $\left(x_{i}{ }^{*}, y_{i}{ }^{*}\right)$ in the place of $\left(x_{i}, y_{i}\right)$ satisfy the condition $\sqrt{u_{i x}{ }^{2}+u_{i y^{2}}}<c c r$, $\forall i \in n$.

\section{Back-tracking Algorithm}

In position based switching, incorporation of back-tracking allows an agent to back-step when the communication topology is not strongly connected. The back-tracking occurs unless the agent reaches the position where communication is regained. At this point the agent takes another path and moves along the consensus point. If an agent is not able to receive data from any of the other agents, it halts and backtracks the path to re-establish the communication link. Then the agent reroutes itself based on algorithmic steps, preserving connectedness of the network. This process is repeated until consensus is reached.

The algorithmic steps are as follows.

1. If $s t e p=1$, the agents broadcast their positions without moving. If $1<$ step $\leq$ step $_{\min }$, the agents broadcast messages to incorporate time-delay and back-tracking in the system.

2. The heading of each agent is updated by the control law $f_{2}\left(u_{i x}, u_{i y}, u_{i v}\right)$ based on feedback from other agents when the agent is not inside consensus circle radius $c c r\left(\sqrt{u_{i x}{ }^{2}+u_{i y}{ }^{2}}>c c r\right)$ with center as average position of other agents.

3. $f_{1}\left(u_{i x}, u_{i y}, u_{i v}\right)= \pm a$ if $\sqrt{u_{i x}{ }^{2}+u_{i y}{ }^{2}}>c c r$ and $u_{i v}<v_{\max }$ i.e., if an agent is not inside the consensus circle radius $c c r$, set acceleration as $\pm a$ until velocity of the agent reaches $v_{\max }$.

4. $f_{2}\left(u_{i x}, u_{i y}, u_{i v}\right)$ continuously updates the heading using obstacle avoidance algorithm in section-5.

5. If an agent is not receiving data from other agents, then $f_{1}\left(u_{i x}, u_{i y}, u_{i v}\right)=-d$ if $u_{i v}>0$ and $f_{1}\left(u_{i x}, u_{i y}, u_{i v}\right)=0$ if $u_{i v}=0$ are used to bring the agent to halt.

6. In the next step, make $f_{1}\left(u_{i x}, u_{i y}, u_{i v}\right)=-a$ to back-track and it is repeated for the next $b_{1}$ number of steps. Then use $f_{1}\left(u_{i x}, u_{i y}, u_{i v}\right)=d$ if $u_{i v}<0$ and $f_{1}\left(u_{i x}, u_{i y}, u_{i v}\right)=0$ if $u_{i v}=0$ to bring the agent comes to halt. 


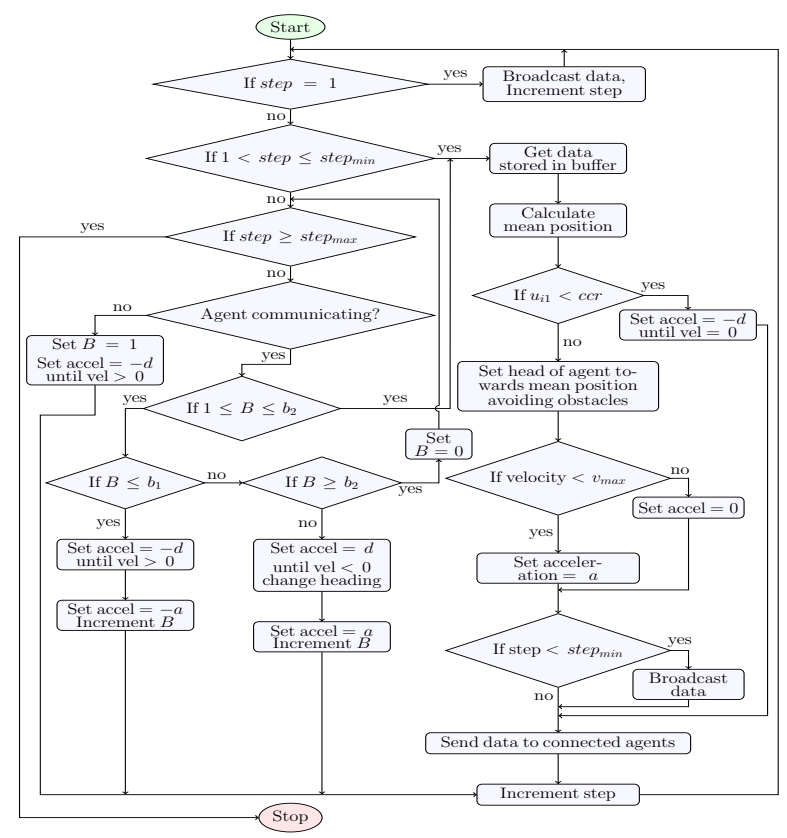

Figure 1: Flow chart for position based switching with back-tracking

7. Change the heading of agent by $\phi_{b}$ degrees using $f_{2}\left(u_{i x}, u_{i y}, u_{i v}\right)$ and make $f_{1}\left(u_{i x}, u_{i y}, u_{i v}\right)=a$ if $\sqrt{u_{i x}{ }^{2}+u_{i y}{ }^{2}}>$ $c c r$ and $u_{i v}<v_{\max }$ for next $b 2$ number of steps.

8. Change heading towards mean position with the help of $f_{2}\left(u_{i x}, u_{i y}, u_{i v}\right)$ and proceed further.

9. Iterate above steps until $\sqrt{u_{i x}{ }^{2}+u_{i y}{ }^{2}}>c c r$

10. When $\sqrt{u_{i x}{ }^{2}+u_{i y}{ }^{2}} \leq c c r$, the agent is brought to halt by making $f_{1}\left(u_{i x}, u_{i y}, u_{i v}\right)=-d$ until $u_{i v}>0$ and $f_{1}\left(u_{i x}, u_{i y}, u_{i v}\right)=0$ if $u_{i v}=0$.

11. The simulation is continued till step $\max$ number of steps.

Parameters $s t e p_{\min }$, step $_{\max }, c c r, a, v_{\max }, b_{1}, b_{2}$ and $\phi_{b}$ are to be judicially chosen for specific applications. The above algorithm is represented using a flow chart in Fig. 1.

\section{History Following with Memory Enabled Agents}

Here, we consider position based switching where agents can retain history of average consensus point. Whenever an agent loses communication with other agents, it proceeds with data stored in memory till it regains communication. The algorithmic steps are very similar to the one without back-tracking except that agent doesn't come to halt when it loses communication. The algorithm can be depicted in a flow chart as given in Fig. 2. Next section presents the obstacle avoidance algorithm to be incorporated with the proposed algorithms.

\section{Sensor Based Obstacle Avoidance}

A laser sensor that is available in MRSim application is used for the purpose of obstacle avoidance. Beam-width of the sensor is defined to be $180^{\circ}$ and a range of 300pixels is assigned. The sensor returns an array of distance values with which we have implemented an algorithm enumerated below. Fig. 3 gives a pictorial representation of the robot shape and area of obstacle avoidance which is divided in a number of sectors. A detailed algorithm is as follows.

1. Scan the $180^{\circ}$ sector in the front using laser scanner to calculate cone of avoidance.

2. If an obstacle is found within 150pixels in the sector of $\pm 2^{\circ}$ of present heading, then turn the agent to nearest $4^{\circ}$ sector with no obstacles.

3. If an obstacle is found within $5 / \sin (\theta)$ distance in the $57^{\circ}$ sectors of $\pm 3^{\circ}$ to $\pm 60^{\circ}$ of present heading, then turn the agent to avoid obstacle with minimum of $1^{\circ}$ change and maximum $30^{\circ}$ change.

4. If an obstacle is found within 8pixels in the 30 sectors of \pm 61 to \pm 90 , then turn the agent by $30^{\circ}$.

5. If an obstacle is found within 5pixels in the sector of $\pm 90^{\circ}$ of present heading, bring the agent to halt position. 


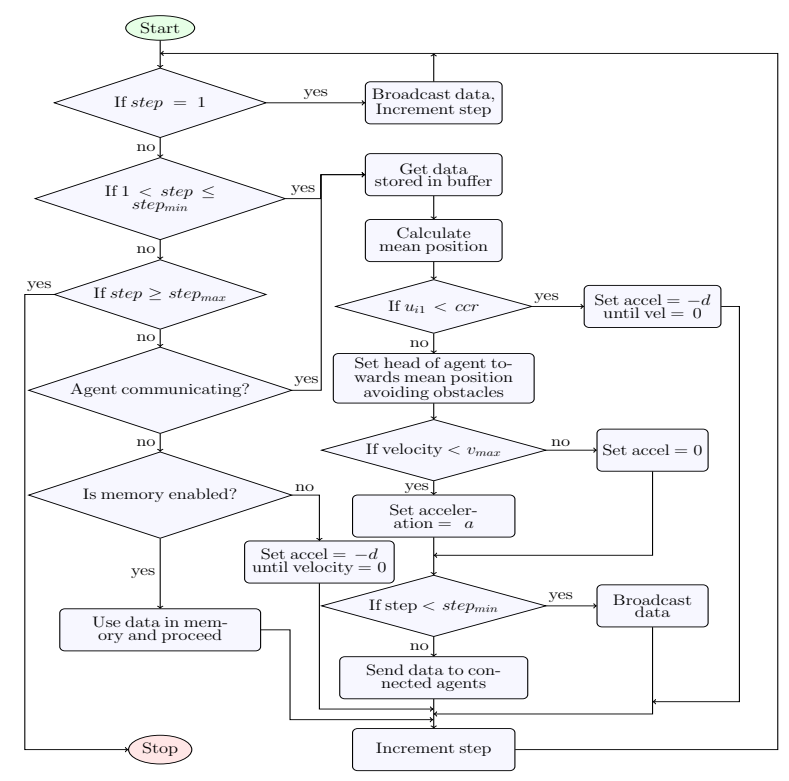

Figure 2: Flow chart for position based switching with and without memory enabled agents

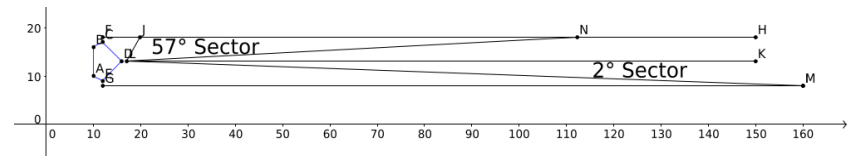

Figure 3: Pictorial Representation of Obstacle Avoidance

\section{Simulation Results}

This section presents the simulation of multi-agent consensus for various non-switching and switching topologies. The simulations are done using MRSim - Multi-Robot Simulator(V1.0) compatible on MATLAB 7.12 (R2011a)+. It can simulate upto 255 robots with user defined shape. The simulation environment is 2-dimensional 1-bit(black/white) bitmap image which considers boarders and obstacles as zero valued pixels and rest of the pixels as value one. The obstacles in simulation results are depicted using darker shade gray boxes and communication loss areas with lighter shade gray boxes.

\subsection{Arena and Initial Conditions}

The arena is chosen to be of size $1412 \times 773 p i x e l s$ whose origin is considered to be at south-west position. The arena has boarders at 26pixels in the West, at 35pixels in the South, at 1384pixels in the East and at 747pixels in the North. Each agent is fit in a circle with 5pixels radius. As there are six agents, consensus circle radius ccr is considered as 30pixels

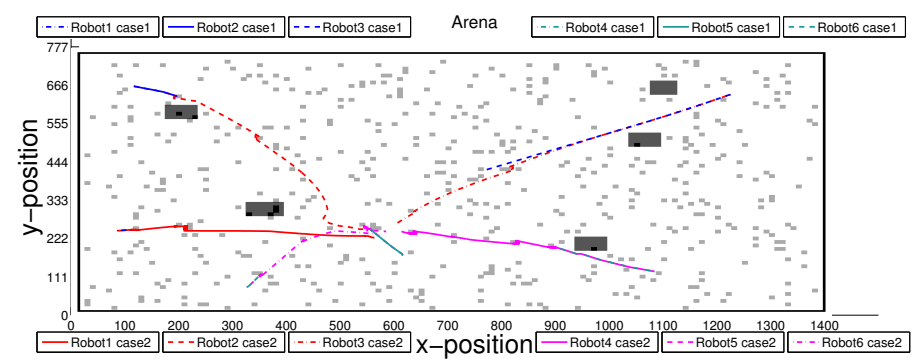

Figure 4: Consensus of switching topologies based on position without back-tracking algorithm(case-1) and with back-tracking algorithm(case-2): loss of communication occurs in $5 \%$ of the total area.

to enable them to fit in the region even if they stand in straight line. The maximum velocity of each agent is considered as $v_{\max }=2$ pixels $/$ step, acceleration as $a=0.1$ pixels $/$ step $^{2}$ and deceleration as $d=0.5$ pixels $/$ step $^{2}$. It is considered 
that for the first $s t e p_{\min }=10$ steps, all the agents broadcast data to enable back-tracking without failure. $s t e p_{\max }$ is considered as 500 for non-switching cases and 800 for position based switching cases. Number of steps for back-tracking is considered as $b_{1}=5$, number of steps for path change is considered as $b_{2}=10$ and change in the angle after back-tracking is considered to be $\phi_{b}=45^{\circ}$.

\subsection{Position Based Switching Communication Topologies}

In practical cases, the position of robot decides the communication topology switching. For the sake of simplicity, we have assumed two communication topologies and the corresponding adjacency matrices are as given below.

$$
A 1=\left[\begin{array}{llllll}
0 & 1 & 0 & 1 & 0 & 1 \\
1 & 0 & 1 & 0 & 1 & 0 \\
0 & 1 & 0 & 1 & 1 & 1 \\
1 & 0 & 1 & 0 & 1 & 0 \\
0 & 1 & 0 & 1 & 0 & 1 \\
1 & 1 & 0 & 1 & 1 & 0
\end{array}\right], A 2=0_{6 \times 6}
$$

The arena will have five percent of area in which the agents lose communication and it is denoted with lighter shade gray boxes in the Fig. 4. Simulation is done using algorithm in Fig. 2 without memory, the agents come to halt when they lose communication, which is shown in Fig. 4 labelled as case-1. The direction robot motion is shown using a green arrow in all the simulation images. Initial positions of robots for result depicted in Fig. 4 are as given below, Robot1: (86, 244), Robot2: (117,663), Robot3: (1225,639), Robot4: (1084, 127), Robot5: (616, 173), Robot6: (326, 80).

\subsection{Position Based Switching Communication Topology with Back-tracking}

Here, we consider switching communication topology based on position with back-tracking by following the algorithm given in section-3. The simulations are performed using the two communication topologies whose adjacency matrices are provided earlier with time delays $g_{j} T \leq 0.6 \mathrm{sec}, g_{i} T=0.1 \mathrm{sec}$. Simulation results depict that whenever an agent is not receiving data, it reroutes using back-tracking algorithm to ensure all agents receive and send data. Simulations are done for two different scenarios as shown in Figs. 4 and 5. The first scenario in Fig. 4 has five percent of area in which the agents lose communication, the second scenario in Fig. 5 has three percent of area in which the agents lose communication denoted by lighter shade gray boxes. Figs. 4 and 5 show the result where agents lose communication at various points but finally reach consensus using back-tracking which is labelled as case-2. Initial positions of robots for result depicted in Fig. 5 are as given below,

Robot1: (943,319), Robot2: (1345, 128), Robot3: $(1312,536)$, Robot4: $(500,616)$, Robot5: (54, 437), Robot6: (194,69)

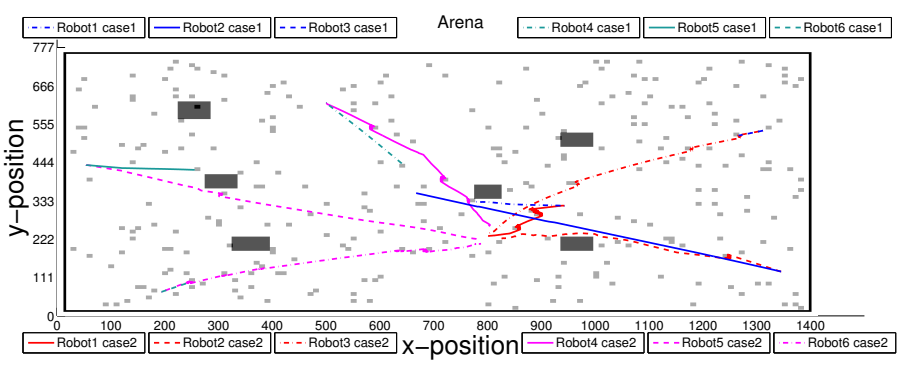

Figure 5: Consensus of switching topologies based on position without back-tracking 20 (case-1) and with back-tracking algorithm(case-2): loss of communication occurs in $3 \%$ of the total area.

\subsection{Position Based Switching Network Topology with Memory Enabled Agents}

In this case, the simulations are performed with the two adjacency matrices as given earlier with time delays $g_{j} T \leq$ $0.6 \mathrm{sec}, g_{i} T=0.1 \mathrm{sec}$. Simulations are done for two different scenarios similar to earlier case as shown in Fig. 6 case-2 and Fig. 7 case-1. From Fig. 6, it can be observed that the agents reach consensus but from Fig. 7 it can be observed that robot1 is not reaching consensus. The reason for this behaviour is, robot1 comes to halt based on data in its memory after losing communication and rest of the agents change path to avoid collision. This is a drawback which arises if an agent couldn't gain communication before rest of the agents reach consensus and come to halt. It can also be observed from Fig. 7 case-2, the agent reach consensus using back-tracking algorithm. Initial positions of robots for result depicted in Fig. 6 are as given below,

Robot1: (86, 244), Robot2: (117,663), Robot3: (1225,639), Robot4: (1084, 127), Robot5: (616, 173), Robot6: (326, 80). 


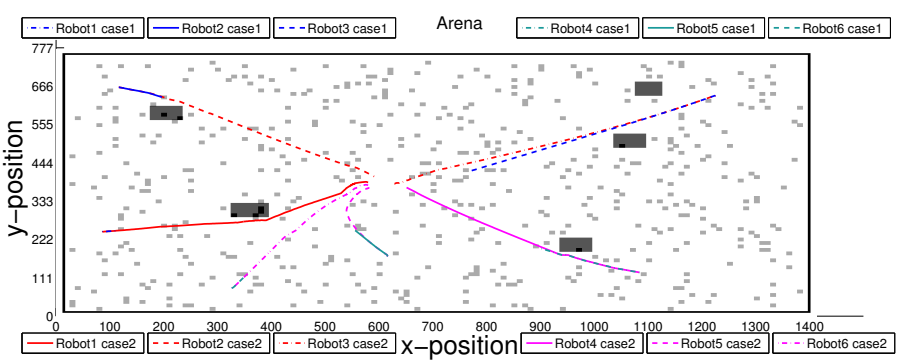

Figure 6: Consensus of switching topologies based on position without memory(case-1) 21] and with memory enabled agents(case2): loss of communication occurs in $5 \%$ of the total area

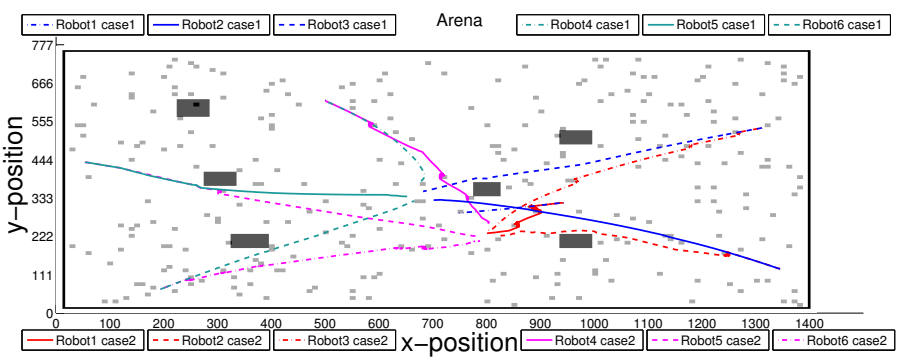

Figure 7: Consensus of switching topologies based on position with memory enabled agents(case-1) and with back-tracking(case-2): loss of communication occurs in $3 \%$ of the total area

Initial positions of robots for result depicted in Fig. 7 are as given below, Robot1: (943,319), Robot2: (1345, 128), Robot3: (1312, 536), Robot4: (500,616), Robot5: $(54,437)$, Robot6: (194,69).

\subsection{Comparison of Results}

In the comparison shown in Table 1, different cases have the following parameters. Case 1: Position based switching without back tracking using algorithm in Fig. 2 without memory. Case 2: Position based switching without back tracking and memory using control laws in 20] 21]. Case 3: Position based switching with back tracking using algorithm in Fig. 1. Case 4: Position based switching with history following using algorithm in Fig. 2. Case A: $a=0.05, d=0.25$, $V_{\min }=-1, V_{\max }=1, c c r=30$, step $_{\min }=20, b_{1}=20, b_{2}=20$ and arena as shown in Fig. 6 . Case B: $a=0.1, d=0.5$, $V_{\min }=-2, V_{\max }=2, c c r=50$, step $_{\min }=10, b_{1}=10, b_{2}=20$ and arena is as shown in Fig. 7. Case C: $a=0.3$, $d=1, V_{\min }=-3, V_{\max }=3, c c r=40$, step $_{\min }=5, b_{1}=5, b_{2}=15$ and arena as shown in Fig. $6 . A-1, B-1, C-1: 3 \%$ communication loss, $A$-2, B-2, $C$-2: $5 \%$ communication loss.

Table 1: A comparison on the time to reach consensus for various switching topologies

\begin{tabular}{|c|c|c|c|c|c|c|}
\hline \multirow{3}{*}{$\begin{array}{l}\text { Switching } \\
\text { topology }\end{array}$} & \multicolumn{6}{|c|}{ Time(sec) to reach consensus } \\
\hline & \multicolumn{2}{|c|}{ Case $A$} & \multicolumn{2}{|c|}{ Case $B$} & \multicolumn{2}{|c|}{ Case $C$} \\
\hline & $A-1$ & $A-2$ & $B-1$ & $B-2$ & $C-1$ & $C-2$ \\
\hline Case 1 & - & - & - & - & - & \\
\hline Case 2 & - & - & - & - & - & \\
\hline Case 3 & 187.6 & 248.4 & 96.6 & 98.2 & 60.4 & 73.2 \\
\hline Case 4 & 133 & 133 & 68.4 & 68.4 & 41.8 & 41.8 \\
\hline
\end{tabular}

From the simulation results shown in Table 1, it is observed that increase in acceleration and velocity with back tracking will not directly reflect in reduced convergence time. The convergence time in a given arena is affected by initial conditions for different arenas and number of times back tracking take place for the last robot while reaching consensus. The number of times back tracked by last robot is different for all the cases, case $A: 8$ and 13 , case B: 5 and 9 , case $C$ : 6 and 7 for $3 \%$ and $5 \%$ loss of communication respectively. Values of $s t e p_{\min }, b_{1}$ and $b_{2}$ will affect the path of robot which will further affect convergence time. All the parameters should be chosen judiciously depending on size of arena, size of no communication zones and size of robots. Proposed algorithms display enough robustness to work for different set of parameters and arena conditions. As expected, observations reveal that the convergence time increases when the percentage of area with no communication increases. In the case of history following algorithm, agents are required to 
store the most recent update and the extra memory required is negligible. The average consensus reaching time is less in case of memory enabled agents, changes in acceleration and velocity reflect in convergence time. However, as shown in Fig. 7, in some situations, following previous history may result in some error in the final destination. We should chose an algorithm considering the trade-offs between convergence time and guaranteed convergence.

We have considered other control laws [20, 21, 22 and the result of simulation is similar as the case without backtracking. The authors in their control laws did not consider the complete loss of communication based on position of robots, the robots will not reach consensus once they lose communication in our arena. The resulting simulation for the control law proposed by Zhou. et al. 20 is shown in Fig. 5 labelled as case 1, we can observe that the robots stop when they lose communication from other robots. Control laws with saturation is considered since the algorithms we proposed also have acceleration and velocity constraints which are similar to saturation. Most of the consensus control laws in literature are linear and switching topologies are periodic in nature. A linear control law will result in large acceleration and velocity when the agents are far from each other, those control laws cannot be used directly in our simulation since obstacle avoidance is designed for low velocity scenario and also due to limitations in hardware.

\section{Hardware Implementation Results}

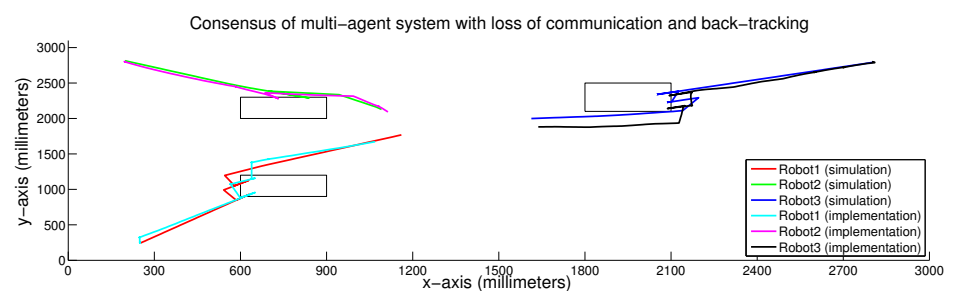

Figure 8: Position of robots in a two dimensional x-y plane

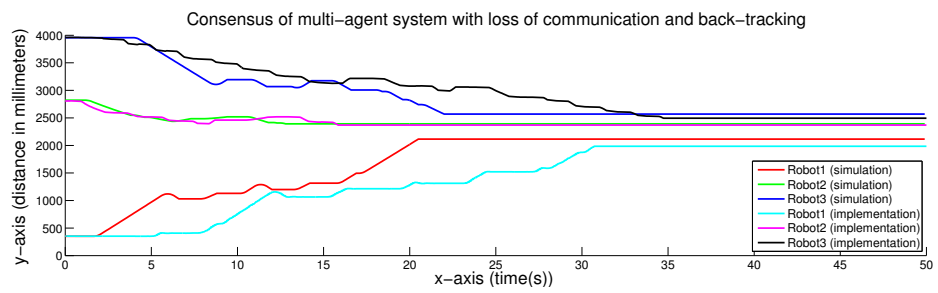

Figure 9: Distance of robots from origin with respect to time

Hardware implementation is done on a network of three agents, one is a Research PatrolBot manufactured by Adept MobileRobots and other two robots powered by BeagleBone-Black are developed in lab. The algorithm implemented on the system is very similar to simulation except the obstacle avoidance part.

We also simulated the three agent system on MRSim for comparison of results. The simulation and implementation results for back-tracking case are as depicted in Figs. 8 and 9. The arena is chosen to be of size $3000 \times 3000$ pixels whose origin is considered to be at south-west position where pixels in simulation are analogous to millimetres in implementation. Fig. 8 shows a case with robots having initial positions $(250,250),(200,2800),(2800,2800)$ and initial angle $0^{\circ}$. For simulation, the values of acceleration and deceleration are chosen to be 50pixels $/ \mathrm{s}^{2}$ and reference velocity to be 200pixels $/ \mathrm{s}$. The values of $b_{1}=5, b_{2}=10$ and $\phi_{b}=45^{\circ}$ are chosen similar to previous simulations. For implementation, acceleration and deceleration are dynamic in nature based on PID controller output. PID controller tires to maintain a reference velocity magnitude of $200 \mathrm{~mm} / \mathrm{s}$ in both forward and backward directions.

Fig. 9 depicts robots' distances from origin $(0,0)$ versus time. The rectangular boxes show the area where robots lose communication. The robots use back-tracking algorithm when they enter into that region to change its path for reaching destination. It is observed that there is some deviation in path followed and difference in convergence time between simulation and implementation. The path deviation is due to errors in differential drive and corresponding PID controller trying to maintain reference velocity of $200 \mathrm{~mm} / \mathrm{s}$. These error is also due to more number of robot heading corrections which increased convergence time.

The simulation and implementation results for memory enabled agents case are shown in Figs. 10 and 11 with same initial conditions and arena. It can be observed that time taken to reach consensus in this scenario is less than that of back-tracking. 


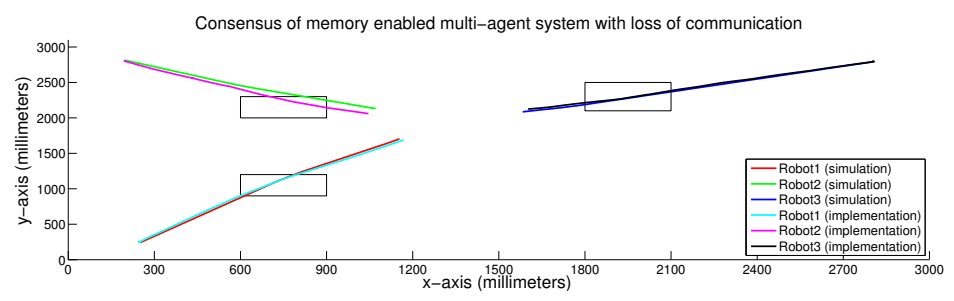

Figure 10: Position of robots in a two dimensional x-y plane

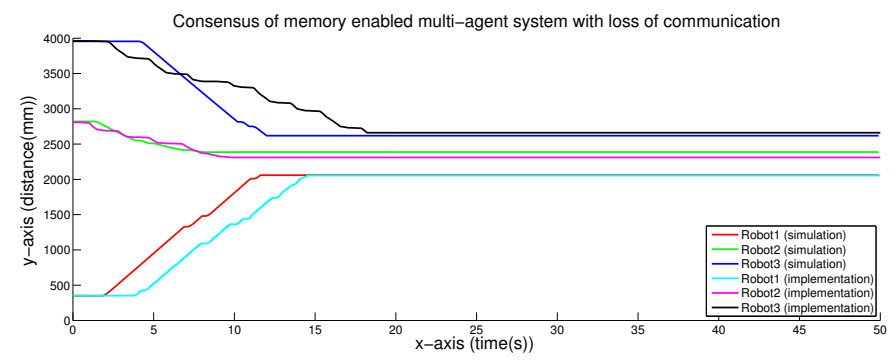

Figure 11: Distance of robots from origin with respect to time

\section{Conclusion}

Two new algorithms, to reach consensus in a multi-agent network with position based switching topologies, are proposed in this work. Each agent is represented by a second order state space model where the control input is computed using the presented algorithms. A number of static obstacles are assumed to be present in the arena. A sensor based obstacle avoidance algorithm ensures no collision between the agents and the obstacles. The switching occurs depending on agents' position. The algorithms guarantee consensus by letting the agents to either back-track take take a different path or follow the previous consensus point whenever a loss of communication takes place. Simulation results show that when the agents back-track and reroute whenever they lose communication with the network, consensus is reached all the time. However, when they follow the previous history, situations may arise where some of the agents stop at locations near the actual consensus point. Otherwise, the time to reach consensus is less in case of history following with memory enabled agents. Hardware implementation without obstacles is also presented which shows the usefulness of the algorithms for practical purposes.

\section{Acknowledgement}

This work is an outcome of the project "Navigation and Path Planning of Mobile Robots and Extension to Multi-Agent Systems", funded by the Department of Science and Technology, Government of India.

\section{References}

[1] T. Vicsek, A. Czirók, E. Ben-Jacob, I. Cohen, and O. Shochet. Novel type of phase transition in a system of self-driven particles. Phys. Rev. Lett., 75:1226, 1995.

[2] E. Garcia, M.A. Jimenez, P.G. De-Santos, and M. Armada. The evolution of robotics research. IEEE Robotics and Automation Magazine, 14:90-103, 2007.

[3] L. Yang, Z. Cao, C. Zhou, L. Cheng, and M. Tan. Formation control and switching for multiple robots in uncertain environments. International Journal of Robotics and Automation, 25(3):240, 2010.

[4] K.S. Senthilkumar and K.K. Bharadwaj. Multi-robot terrain coverage by constructing multiple spanning trees simultaneously. International Journal of Robotics and Automation, 25(3):195, 2010.

[5] Paulo A. Jiménez, Bijan Shirinzadeh, Denny Oetomo, and Ann Nicholson. Swarm aggregation and formation control for robots with limited perception. International Journal of Robotics and Automation, 26(3):255-263, 2011.

[6] R.O. Saber and R.M. Murray. Consensus protocols for networks of dynamic agents. In Proceedings of the American Control Conference, volume 2, pages 951-956, 2003. 
[7] R. O. Saber and R.M. Murray. Consensus problems in networks of agents with switching topology and time-delays. IEEE Trans. on Automatic Control, 49:1520-1533, 2004.

[8] L. Moreau. Stability of continuous-time distributed consensus algorithms. In Decision and Control, CDC, 43rd IEEE Conference, pages 3998-4003, 2004.

[9] R.O. Saber, J.A. Fax, and R.M. Murray. Consensus and cooperation in networked multi-agent systems. Proceedings of the IEEE, 95:215-233, 2007.

[10] W. Ren, K. Moore, and Y. Chen. High-order consensus algorithms in cooperative vehicle systems. In IEEE Int. Conf. on Networking, Sensing and Control, pages 457-462, 2006.

[11] P.-A. Bliman, A. Nedic, and A. Ozdaglar. Rate of convergence for consensus with delays. In Decision and Control, CDC, 47th IEEE Conference, pages 4849-4854, 2008.

[12] J. Hu and Y.S. Lin. Consensus control for multi-agent systems with double-integrator dynamics and time delays. Control Theory and Applications, IET, 4:109-118, 2010.

[13] T. Shida and H. Ohmori. Robustness of consensus algorithm for communication delays and switching topology. In Proceedings of SICE Annual Conference, pages 1373-1380, 2011.

[14] W. He and J. Cao. Consensus control for high-order multi-agent systems. Control Theory and Applications, IET, 5:231-238, 2011.

[15] P. Lin, Z. Li, Y. Jia, and M. Sun. High-order multi-agent consensus with dynamically changing topologies and time-delays. Control Theory and Applications, IET, 5:976-981, 2011.

[16] Y.-H. Chang, C.-W. Chang, C.-L. Chen, and C.-W. Tao. Fuzzy sliding-mode formation control for multirobot systems: Design and implementation. IEEE Trans. on Systems, Man, and Cybernetics, Part B: Cybernetics, 42:444-457, 2012.

[17] Ting Wang, Cristophe Sabourin, and Kurosh Madani. A novel path planning approach for multi-robot based transportation. International Journal of Robotics and Automation, 28(3):218-225, 2013.

[18] Z. Meng, W. Ren, Y. Cao, and Z. You. Leaderless and leader-following consensus with communication and input delays under a directed network topology. IEEE Trans on Systems, Man, and Cybernetics, Part B: Cybernetics, 41:75-88, 2011.

[19] Haopeng Zhang and Qing Hui. Topological heterogeneity and optimality analysis for multiagent formation. In Decision and Control (CDC), 2012 IEEE 51st Annual Conference on, pages 5954 - 5959, 2012.

[20] Shaolei Zhou and Shi Yan. The stability analysis for a class of Multi-Agent group formation with input saturation constraints. In Proceedings of 2014 IEEE Chinese Guidance, Navigation and Control Conference, pages 1618-1623, 2014.

[21] Zhiqiang Zuo, Zhicheng Zhang, and Yijing Wang. Finite-time consensus of double integrator multi-agent systems subject to input saturation. In Chinese Control Conference, CCC, volume 2015-Septe, pages 7280-7284, 2015.

[22] Airong Wei, Xiaoming Hu, and Yuzhen Wang. Tracking Control of Leader-follower Multi-agent Systems Subject to Actuator Saturation. IEEE/CAA JOURNAL OF AUTOMATICA SINICA, 1(1):84-91, 2014. 